Design Systematic review and meta-analysis using individual participant data.

Data sources Individual patient data were obtained from eligible randomised controlled trials found by searching the electronic databases Medline, Embase and the Cochrane Central Register of Controlled Trials and reference lists of all identified articles.

Eligibility criteria for selecting studies Trials were eligible if they included patients with minimally symptomatic OSA, had randomised them to receive CPAP or either sham-CPAP or no CPAP, and had measured blood pressure at baseline and at a follow-up visit.

Results Five eligible trials were found (1219 patients) from which the necessary data from four studies (1206 patients) was obtained. There was some evidence that CPAP treatment was associated with a small increase in systolic blood pressure of $1 \mathrm{mmHg}$ (95\% confidence interval -0.1 to 2.2 ), $\mathrm{p}=0.079$, with a larger increase in those patients using CPAP less than four hours/night $(+2.7 \mathrm{mmHg}, 95 \%$ CI $(1.1$ to 4.2$), \mathrm{p}=0.001)$. There was no overall effect on diastolic blood pressure (DBP), however, there was evidence of a reduction in DBP in patients using CPAP more than four hours/night $(-1.2 \mathrm{mmHg}, 95 \%$ CI (2.1 to -0.3$), p=0.013)$ and an increase in those using it less than four hours/night $(+1.2,95 \%$ CI $(0.1$ to 2.3$), \mathrm{p}=0.035)$. CPAP treatment reduced both subjective sleepiness and OSA severity (both $\mathrm{p}<0.001$ ), with larger reductions in patients using treatment more than four hours/night.

Conclusions Although CPAP treatment reduces OSA severity and sleepiness, it seems not to have a beneficial effect on blood pressure (and possibly a detrimental effect on systolic BP) in patients with minimally symptomatic OSA, unless they use CPAP for more than four hours per night.

\section{S4 SHORT TERM USE OF CONTINUOUS POSITIVE AIRWAY PRESSURE (CPAP) BY OBESE PATIENTS WITH OBSTRUCTIVE SLEEP APNOEA SYNDROME (OSAS) DIAGNOSED DURING ASSESSMENT FOR BARIATRIC SURGERY}

S Thomas, HR Gribbin, G Danjoux, R Sathyamurthy.; Sleep Clinic, Division of Anaesthesia, James Cook University Hospital, Middlesbrough TS4 3BW, UK

\subsection{6/thoraxjnl-2013-204457.10}

Around one in three patients with OSAS is unable to continue with CPAP. The reasons for this are still poorly understood. Patients being assessed for Bariatric Surgery are informed that untreated OSAS can increase peri-operative risk. We investigated whether this information had an effect on CPAP use. Over a six month period we identified 22 obese patients (Mean BMI 49.3) with OSAS discovered during assessment for Bariatric Surgery (OSAS Bar). Over the same period we identified a group of 33 obese patients (Mean BMI 42.1) with OSAS referred through conventional pathways by General Practitioners and Hospital Doctors (OSAS Conv) not under consideration for Bariatric Surgery. OSAS was diagnosed by overnight pulse oximetry, clinical interview and Epworth Sleepiness Score (ESS) using established criteria.

Results There were more women than men in the OSAS Bar group (41\%) than in the OSAS Conv group (18\%). Apart from this the 2 groups (OSAS Bar vs OSAS Conv) were well matched prior to CPAP for: Age (Mean 51.2 vs 48.3, p = ns), >4\% Oxygen Desaturation Index (Mean 31.9/hour vs 42.3/hour, $\mathrm{p}=\mathrm{ns}$ ) and ESS (Mean 12.8 vs $14.8, \mathrm{p}=\mathrm{ns}$ ). By 6 weeks after Out-
Patient CPAP initiation, 6(27\%) of the OSAS Bar group and 7 (21\%) of the OSAS Conv group had stopped using CPAP. In the patients continuing with CPAP there was no difference between the two groups in hours of CPAP used per night as measured from the CPAP device clock: CPAP Bar (Mean 5, Range 1-8, hours), CPAP Conv (Mean 6, Range 1.5-8.5, hours) or in the ESS on CPAP: CPAP Bar (Mean ESS 6.8), CPAP Conv (Mean ESS 5.8).

Conclusion This group of obese patients with OSAS under assessment for Bariatric Surgery had similar CPAP use to obese patients with OSAS not considering surgical intervention. Information on peri-operative risk does not appear to be a motivator in overcoming whatever inhibiting factors prevent patients from using CPAP. Pre-operative assessment clinics should be aware that CPAP prescription does not necessarily mean that CPAP is being used.

\section{S5 VARIABILITY IN CLINICIAN'S PERCEPTION REGARDING FITNESS TO DRIVE IN PATIENTS WITH OBSTRUCTIVE SLEEP APNOEA SYNDROME (OSAS)- ON BEHALF OF THE BRITISH THORACIC SOCIETY SLEEP APNOEA SAG.}

${ }^{1}$ A Dwarakanath, ${ }^{1} D$ Ghosh, ${ }^{2} S L$ Jamson, ${ }^{3}$ PD Baxter, ${ }^{4} \mathrm{M}$ Twiddy, ${ }^{1} \mathrm{MW}$ Elliott.; ${ }^{2}$ St. James University Hospital, Leeds, United Kingdom; ${ }^{2}$ Institute for Transport Studies, University of Leeds, Leeds, United Kingdom; ${ }^{3}$ Division of Biostatistics, LIGHT, Centre for Epidemiology and Biostatistics, University of Leeds, Leeds, United Kingdom; ${ }^{4}$ Leeds Institute of Health Sciences, Leeds, United Kingdom

\subsection{6/thoraxjnl-2013-204457.11}

Introduction Advice about driving is a key component of the management of OSAS patients. No objective tests have been shown to predict reliably whether an individual is safe to drive or not and therefore the advice given will depend upon the opinion of clinicians. We evaluated the current practice of advice given regarding fitness to drive in OSAS patients.

Methods Clinicians were invited to participate in a web-based survey. The questionnaire included six clinical vignettes describing a variety of OSAS patients. For each the respondent chose from options ranging from driving without restriction to advising not to drive at all. For ease of presentation the data are summarised as whether would allow driving or not.

Results 467 respondents completed the survey. The advice given by the respondents to various clinical vignettes was variable (figure-1). In the least contentious scenario (vignette-1) there was 1 in 14.6 chance and in the most (vignette-4) there was a 1 in 2 chance of an individual being told whether they could drive or not. Respondents were more likely to advise patients to refrain from driving if the AHI was worse (P- $<0.0001$, OR-3.9), if the Epworth sleepiness score was high $(\mathrm{P}-<0.0001, \mathrm{OR}-23.5)$ and if

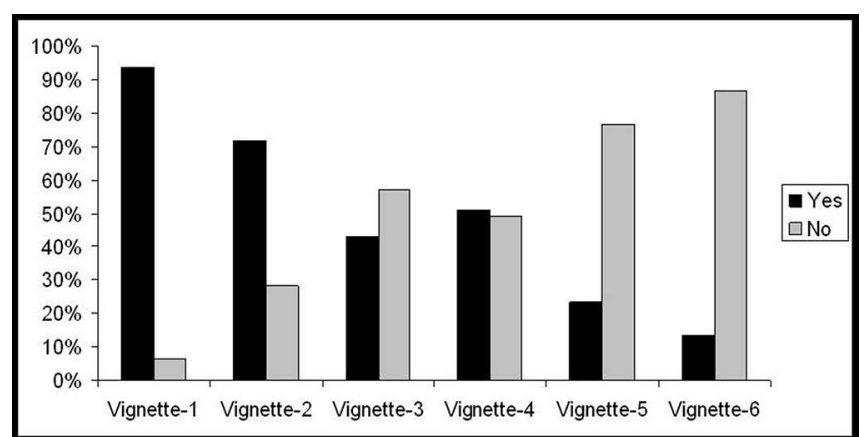

Abstract S5 Figure 1. 
the patient admitted to any problems at all while driving ( $\mathrm{P}$ 0.0098, OR-1.6). Males were more likely to allow OSAS patients to drive. Consultants without an interest in sleep medicine and respondents seeing less than 5 patients per month were more likely to advise against driving. The advice given was not related to the age of the clinician.

Conclusions This survey has shown that there is considerable variability in the advice given by clinicians, with in some situations a patient having an even chance of receiving opposite advice, depending on who they see. Restriction of driving has major implications for an individual, both social and financial. Allowing someone to drive who is not safe to do so has potentially disastrous consequences for them and others. The issue of how to improve consistency of advice needs to be addressed.

\section{Non-invasive markers of lung disease in children}

\section{S6 LONG TERM LUNG FUNCTION OUTCOMES IN CHILDREN BORN WITH ABDOMINAL WALL DEFECTS}

${ }^{1} \mathrm{~S}$ Rajalingam, ${ }^{2} \mathrm{Q}$ Mok, ${ }^{2} \mathrm{C}$ Curry, ${ }^{3}$ Eaton, ${ }^{2} \mathrm{E}$ Fettes, ${ }^{3} \mathrm{~J}$ Kirkby, ${ }^{2} \mathrm{P}$ Aurora; ${ }^{1}$ Newcastle University, Newcastle, United Kingdom; ${ }^{2}$ Great Ormond St Children's Hospital, London, United Kingdom; 'Institute of Child Health, University College London, London, United Kingdom

\subsection{6/thoraxjn-2013-204457.12}

Introduction Although survival following surgical repair of abdominal wall defect is now good, there are very few data regarding long-term lung function (LF) outcomes. The aim of this study was to describe LF at school-age for a post-surgery population from a single large centre.

Methods All children having surgical repair of exomphalos or gastroschisis between 1/1/1998-30/6/2007 at our institution were identified, and those aged greater than 6 years were recalled for LF testing. Children born with major associated cardiac, genetic or laryngeal conditions were excluded from the study. All children underwent resting oximetry, body plethysmography, forced spirometry and carbon monoxide transfer test. Clinical and demographic data was also collected.

Results were converted to z-scores to adjust for height, sex, age and ethnicity. Statistical comparisons between groups were performed with t-tests.

Results 86 children were approached and LF data were obtained in 31 (12 Exomphalos;19 Gastroschisis) children aged $\geq 6$ years. 22 children were male; the mean age at testing was 9.9 years (range: 6.4-14.4). When analysed as a group these children had significantly reduced forced expiratory volumes in $1 \mathrm{~s}, \mathrm{FEV}_{1}(-0.52 \mathrm{z}-$ scores [95\%CI -1.01,-0.04] $\mathrm{p}=0.036), \mathrm{FEV}_{1} / \mathrm{FVC}(-0.88$ [-1.21, $0.56] \mathrm{p}<0.000)$, lung transfer factor, $\mathrm{TL}_{\mathrm{CO}}(-0.76[-1.05,-0.47]$ $\mathrm{p}<0.000), \mathrm{TL}_{\mathrm{CO}} / \mathrm{VA}(-1.77[-2.08,-1.46] \mathrm{p}<0.000)$, functional residual capacity, FRC $(-0.45[-0.70,-0.19] \mathrm{p}=0.001)$, residual volume, RV $(-0.36[-0.61,-0.12] \mathrm{p}=0.006)$ and significantly increased alveolar volume, VA $(0.95[0.37,1.53] \mathrm{p}=0.002)$. Forced vital capacity, FVC was normal $(-0.01[-0.54,0.51] \mathrm{p}=0.96)$

When analysed as subgroups, children post exomphalos repair had significantly lower LF than those post gastroschisis repair, with a mean difference $(95 \% \mathrm{CI}$ of difference [Exomphalos-Gastroschisis] ) in $\mathrm{FEV}_{1}$ of $-1.52 \mathrm{z}$-scores $(-2.35,-0.68 ; \mathrm{p}=0.001)$; FVC -1.54 (-2.47, -0.62; p = 0.002); TLC -0.91 (-1.78, -0.04; $\mathrm{p}=0.041)$. No significant group differences were found in the remaining lung function outcomes.
Conclusions This is the largest study to report lung function at school-age in children post abdominal wall surgery. We demonstrate that these children have significantly reduced lung function; and when analysed by subgroup, that those with a history of exomphalos repair have significantly greater defect than those with a history of gastroschisis repair.
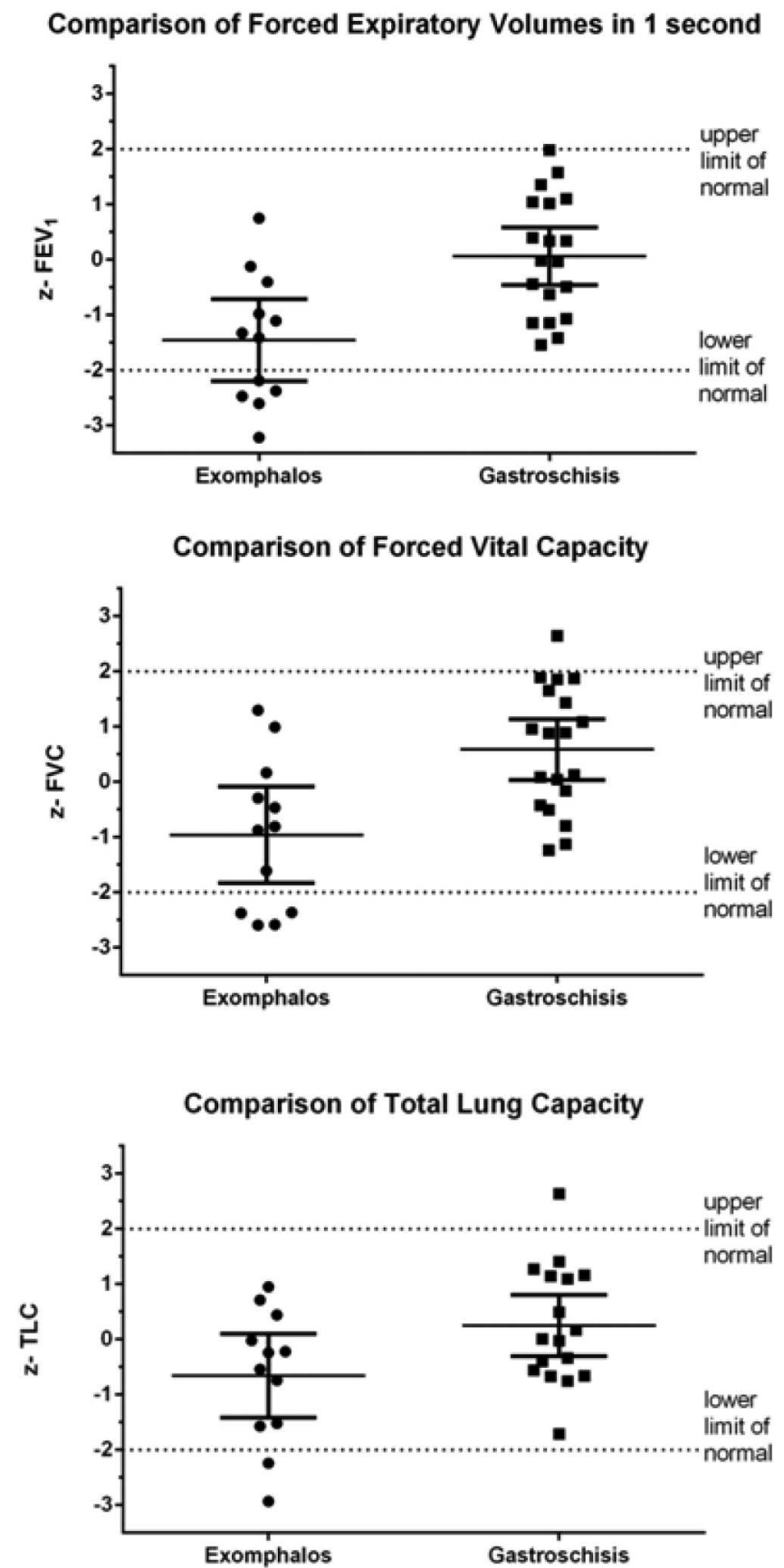

Abstract S6 Figure 1. Comparison of lung function outcomes between groups. Spirometry and plethysmography $z$-scores derived from Global Lung Function Initiative 2012 and Rosenthal 1993 equations respectively.

\section{S7 EVOLUTION OF LUNG FUNCTION DURING THE FIRST TWO YEARS OF LIFE IN INFANTS WITH CYSTIC FIBROSIS DIAGNOSED BY NEWBORN SCREENING}

${ }^{1} \mathrm{~L}$ C Brennan, ${ }^{1} \mathrm{~L}$ P Thia, ${ }^{1} \mathrm{~A}$ Hoo, ${ }^{1} \mathrm{~T}$ Nguyen, ${ }^{1} \mathrm{~J}$ Chudleigh, ${ }^{1} \mathrm{~S}$ Lum, ${ }^{2} \mathrm{~A}$ Wade, ${ }^{3} \mathrm{C}$ Wallis, ${ }^{4} \mathrm{~A}$ Bush, ${ }^{4} \mathrm{I}$ Balfour-Lynn, ${ }^{5} \mathrm{H}$ Wyatt, ${ }^{4} \mathrm{~S}$ Carr, ${ }^{1} \mathrm{~J}$ Stocks; ${ }^{1}$ Portex Respiratory Unit, UCL 